\title{
Análisis de la Justicia Restaurativa en Materia de Responsabilidad Penal para Adolescentes en Colombia*
}

\section{Analysis of Restorative Justice in Criminal Liability for Adolescents in Colombia}

\author{
Leonardo Alberto Rodríguez Cely \\ Pontificia Universidad Javeriana, Colombia
}

\begin{abstract}
Resumen. Este estudio tuvo como objetivo hacer un análisis de la justicia restaurativa orientado a la atención de casos de responsabilidad penal para adolescentes infractores colombianos de acuerdo con la Ley 1098 de 2006. Este análisis es el producto de un trabajo conceptual y un ejercicio metodológico aplicado a un proceso de recolección, sistematización y análisis de la información, basado en un diseño de investigación exploratoria. Se utilizó una muestra intencional de treinta participantes que trabajan en el Centro de Servicios Jurídicos Especiales para Adolescentes, integrada por jueces de conocimiento y de control de garantías, fiscales delegados, defensores de familia, policía judicial, psicólogas y trabajadoras sociales, a quienes se les administró una entrevista en profundidad basada en categorías previamente definidas. En el estudio se describen necesidades del contexto institucional, profesional y de los funcionarios del sistema, que permiten comprender las condiciones necesarias para proponer lineamientos generales que guíen, por una parte, la formulación de un programa de justicia restaurativa acorde con la ley vigente en Colombia y, por otra, que posibiliten diferentes estrategias para empoderar a la comunidad y cumplir el principio de corresponsabilidad entre el Estado, la comunidad, la familia, las instituciones educativas, las víctimas y los adolescentes infractores, para hacer viables los programas de justicia restaurativa.

Palabras clave: adolescentes, justicia restaurativa, responsabilidad penal.
\end{abstract}

\begin{abstract}
This study aimed to analyze the restorative justice oriented to dealing with cases of criminal responsibility for Colombian young offenders under Law 1098 of 2006. This analysis is the product of a conceptual job and methodological exercise applied to a collection process, systematization and analysis of information, based on an exploratory research design. We used a purposive sample of thirty participants who work at the Center for Special Legal Services for Adolescents, composed of judges of knowledge and control of guarantees, prosecutors, family advocates, judicial police, psychologists and social workers who are given an in-depth interview based on predefined categories. In the study are described needs of the institutional context, professional and system staff members that provide insight into the necessary conditions to propose general guidelines to guide on the one hand, the development of a restorative justice program in a accordance with applicable law in Colombia and, on the other hand, that allow different strategies to empower the community and fulfill the
\end{abstract}

La correspondencia sobre este artículo debe enviarse al autor al email:leocely@gmail.com
* Investigación para obtener el Diplomado de Estudios Avanzados (DEA) del programa de Doctorado en Criminología y Delincuencia Juvenil. Agradecimiento por el apoyo académico al Instituto de Criminología de la Universidad de Castilla La Mancha, a la tutora de la investigación Dra. Esther Fernández Molina. Subvencionado por la Pontificia Universidad Javeriana. Colombia. 
principle of shared responsibility between government, community, family, educational institutions, victims and young offenders to make restorative justice programs viable.

Keywords: adolescents, restorative, criminal responsibility.

La justicia restaurativa se gestó a partir de la compresión de las formas como se solucionaban conflictos en las antiguas comunidades indígenas de Nueva Zelanda, Irlanda, Canadá, Estados Unidos y América Latina, cuyas prácticas se han estudiado y retomado en tanto representan una alternativa de justicia que ha permitido el desarrollo de importantes tratados y convenios en los diferentes sistemas de justicia penal de menores en el mundo. Entre ellos se encuentra el de Colombia, que vive un proceso de transición de un sistema eminentemente tutelar a un sistema de justicia o responsabilidad penal juvenil (Griffiths y Hamilton, 1996; Pratt, 1996; Varona, 1998; Consedine 2002; Brito, 2009).

La Convención de los Derechos del Niño CDN- (1989) ha marcado una pauta a través de la propuesta de principios e instrumentos internacionales que prácticamente todos los Estados miembros de la CDN han tomado en cuenta al formular legislaciones especiales reguladoras de la responsabilidad penal de los menores de edad. Una muestra de ello es que los sistemas actuales de justicia juvenil han venido incorporando principios de justicia restaurativa y del modelo de descriminalización, desjudicialización, diversificación y debido proceso, en el marco de procedimientos garantistas y respetuosos de los derechos de los menores de edad (De la Cuesta 1998; Giménez, 1999; Tiffer, 2000; Carranza, Tiffer y Maxera, 2002; Padilla 2010).

En esta vía se ha venido configurando una nueva política criminal de justicia penal juvenil de contenido sancionador-educativo que tiene en cuenta los principios de oportunidad e intervención mínima y que incorpora mecanismos como la mediación reparadora entre víctima e infractor, lo que se vincula, en ocasiones, a medidas de orden comunitario (Rodríguez, Padilla, Rodríguez y Díaz, 2010). El desarrollo de estos procedimientos reconoce a los menores de edad como sujetos de derechos y de responsabilidad y, además, implica características como la especificidad de un derecho penal de menores que aboga por procesos garantistas, flexibles, sumarios, únicos y confidenciales. De esta forma, la acción penal incorpora un amplio cuadro de medidas y sanciones que tiene presentes los principios de oportunidad ${ }^{1}$, intervención mínima ${ }^{2}$ y subsidiariedad $^{3} \quad$ (Tiffer, 2000; ONU, 2002; Larrauri, 2004; Von Hirsch; Ashworth y Shearing, 2003).

Gracias a este nuevo marco de política criminal en Colombia, se hace necesario comenzar con el diseño de criterios para programas de justicia restaurativa en materia de responsabilidad penal para adolescentes, a partir de la ley 1098/2006. Para tal fin, en esta investigación se indaga sobre los orígenes, los principios filosóficos, los valores, los procedimientos y los alcances de esta forma de justicia, así como también por los valores comunitarios y los lineamientos nacionales e internacionales que subyacen la puesta en marcha de programas propios de esta justicia (Carranza, Tiffer, Maxera, 2002; Álvarez, 1999; Álvarez, Corzo, Mendoza, Parra y Rodríguez, 2008).

El artículo presenta en su última parte los resultados de un ejercicio metodológico de corte cualitativo que ofrece lineamientos para un programa piloto de justicia restaurativa dirigido a atender casos de Responsabilidad Penal para Adolescentes en el Centro de Servicios Jurídicos Especiales para Adolescentes (en adelante CESPA) en la ciudad de Bogotá y municipios de Cundinamarca (Colombia).

\footnotetext{
1 "Impone la creación de unas causales que bajo la idea del derecho penal mínimo, de su carácter fragmentario y de última ratio, permitan interrumpir, suspender y renunciar de la acción penal, de paso esta solución conlleva a un menor costo social; al permitirse así sea por excepción que se pueda disponer de la persecución penal” (Fiscalía General de Nación, 2007).

2 "El derecho penal no interviene de cara a la regulación de todos los comportamientos del hombre en sociedad, sino sólo en orden de evitar los atentados más graves que se dirijan contra importantes bienes jurídicos" (Villegas, 2009).

3 "En virtud de la subsidiariedad, todos los niveles de la organización social de orden superior y más complejo se ponen en una actitud de ayuda (palabra latina: subsidium) -apoyo, promoción, desarrollorespecto a los menores" (Gómez, 2008).
} 


\section{Situación de los niños, niñas y adolescentes infractores de la ley en Colombia}

El miedo y la desesperanza se apoderan cada día más de miles de ciudadanos y ciudadanas que viven en la ciudad de Bogotá y en municipios de Cundinamarca cuando tiene que transitar por algunas localidades especialmente peligrosas por las bandas de niños, niñas y adolescentes que operan en ellas (Bosa, Ciudad Bolívar, los Mártires, la Candelaria y Kennedy). De acuerdo con el CESPA, cada día se presenta una cantidad alarmante de delitos como homicidio, lesiones personales, extorsión, amenaza, hurto, robo, asaltos sexuales y micro-tráfico en los que están involucrados menores de edad que infringen la ley penal, lo que representa un grave problema con serias repercusiones en el ámbito social, comunitario, educativo, familiar y personal, que deja un creciente número de víctimas y una preocupante tasa de reincidencia de los menores infractores, que viene en ascenso en los últimos años en la ciudad de Bogotá (Fundación Restrepo Barco, 2004; García, 2006; Márquez, 2007; Restrepo, 2007; Rivera y Barreto, 2008; ONU, 2009).

Lamentablemente, muchos de estos niños, niñas y adolescentes están siendo reclutados sistemáticamente por la delincuencia común, por la delincuencia organizada y otro tanto por antiguos ex militantes de las guerrillas y paramilitares que amparados bajo el programa de reinsertados y desmovilizados de la ley 975 de Justicia y Paz de 2005 siguen delinquiendo y utilizando a menores de edad para cometer diferentes delitos. Bajo el pretexto de que los menores van a recibir grandes sumas de dinero y que obtendrán reconocimiento dentro del grupo, les ofrecen además protección a los miembros de su familia, pero sobre todo hacen un proceso de convencimiento respecto a los beneficios e impunidad que otorga el código de infancia y adolescencia por el carácter pedagógico, flexible y laxo de la norma y sus desbordantes garantías para los menores infractores (Ley 1098/2006; García 2006; Márquez, 2007; ONU, 2009).

Esta situación ha hecho que entre los ciudadanos y ciudadanas cada día tome más fuerza un argumento: que se han disparado las estadísticas de los delitos cometidos por los adolescentes, gracias a la inoperancia del Estado y la falta de control del apa- rato judicial. A esto se suma el miedo de los ciudadanos que recurrentemente han sido víctimas de delitos, muchos de los cuales no se atreven a denunciar por el miedo, la amenaza y la poca credibilidad que genera el sistema. Cada vez se hace más eco a la idea que a partir de la entrada en vigencia de la ley 1098 de 2006 se ha desbordado la acción delictiva de las bandas, que en su mayoría son conformadas por niños, niñas y adolescentes, bajo la dirección de criminales adultos (García 2006; Márquez, 2007; Rivera y Barreto, 2008).

Para describir quiénes son los adolescentes infractores en Colombia, se hace necesario caracterizarlos en condiciones propias del contexto y, por ende, de su vulnerabilidad. Se puede mencionar que existe un dramático cuadro de miseria de niños, niñas y adolescentes con problemas de desnutrición, según el último estudio de desnutrición que se conoce en el país, realizado por el Instituto Colombiano de Bienestar Familiar (en adelante ICBF) (2010), la tasa de prevalencia en la población infantil del país es del $12 \%$. Esto quiere decir que de cada cien niños colombianos 12 presentan desnutrición crónica.

Este estudio señala además que un $13 \%$ de los niños en edades entre los cinco y nueve años tienen algún retraso en su crecimiento y el $5 \%$ tiene un peso bajo para su edad. El informe establece también que de la población entre los 10 y los 17 años un $16 \%$ tiene baja estatura y el $7 \%$ tiene un peso demasiado bajo (ICBF, 2010).

A las problemáticas de desnutrición y mendicidad, se suma la deserción escolar de niños, niñas y adolescentes que de acuerdo con los datos del Ministerio de Educación Nacional (2009), representa el $5.4 \%$. Otro problema que afecta a la niñez y adolescencia en Colombia es la desintegración familiar, la farmacodependencia y la delincuencia vinculada a diferentes grupos armados que utilizan actualmente menores de edad y cuyas cifras las exponen la Defensoría del Pueblo y UNICEF (2006) quienes calculan que hoy hay más de 7.000 niños y niñas en las filas de los grupos armados irregulares. De ellos, el $60 \%$ ha visto matar; el $78 \%$ ha estado frente a cadáveres mutilados; el $25 \%$ ha visto secuestrar; el $13 \%$ ha secuestrado; el $18 \%$ ha presenciado torturas; el $40 \%$ ha disparado contra alguien y el $28 \%$ ha sufrido heridas. 
Adicionalmente, de acuerdo con el reporte de la Fundación Restrepo Barco (2004), de todos los niños y las niñas que participan en hostilidades armadas en el mundo, el $12 \%$ son colombianos que participan en la guerrilla, los grupos de autodefensas, narcotraficantes, grupos de delincuencia organizada y grupos de sicarios que se han vuelto a activar de forma importante después de la década de los ochenta con los grandes carteles de las drogas.

El ICBF, las Comisarias de Familia, el CESPA, la Policía Nacional, la Defensoría del Pueblo denuncian, por su parte, las tasas bajas de denuncias e impunidad (Rivera y Barreto, 2008) y, a la vez, muestran una vinculación alarmante de menores de edad que participan en delitos. El sistema de información estadístico delincuencial, operativo y contravencional de la Policía Nacional (en adelante SIEDCO) muestra una alta participación de niños, niñas y adolescentes como víctimas de homicidios, que entre los años 2002 y 2009 han alcanzado un total de 15.817 participaciones. En cuanto a lesiones la cifra alcanza los 35.324 menores de edad. En el caso del delito de hurto de bienes a los menores, del 2008 al 2009 hubo un incremento del 41\% (1.682 casos más respecto al año anterior). Pero los delitos que presentan mayor participación comparado con población adulta son aquellos contra la libertad, la integridad y la formación sexuales, pues oscilan entre el $70 \%$ y el $76 \%$ entre el 2003 al 2009. Además de esta caracterización es importante recordar que muchos de los y las adolescentes en Colombia son el producto de una historia reciente de padres y familias que han nacido en condiciones de pobreza (Restrepo, 2007) y con una historia social y cultural de un conflicto armado que dura ya más de sesenta años en este país y que se ha caracterizado entre otros aspectos por: a) El problema de valores, normas y creencias sociales que ha dejado la cultura del narcotráfico, especialmente en la década de los años ochenta y noventa; b) muchos de los niños, niñas y adolescentes en Colombia han vivido el desplazamiento forzado al lado de sus familias, que actualmente conforman los cordones de miseria de las principales ciudades del país como consecuencia del accionar bélico de los grupos guerrilleros, en especial las Fuerzas Armadas Revolucionarias de Colombia (FARC); c) el secuestro de los niños, niñas y adolescentes se convirtió en la década de los años 90 y comienzos de 2000 en una estrategia de guerra, forma de terrorismo y soporte económico, dirigido a civiles nacionales y en algunos casos a extranjeros; d) la cantidad de menores de edad que han sido mutilados por las minas antipersonales; e) la utilización de adolescentes para actividades delictivas como el homicidio, la extorsión, el secuestro, el hurto, el tráfico de drogas; f) adolescentes víctimas de los falsos positivos ${ }^{4}$ por parte de la fuerzas armadas que reflejan una parte del conflicto interno colombiano y g) fleteo.

Para contrarrestar los anteriores delitos, infortunadamente se cuenta con un modelo de justicia en crisis y con un grave problema de impunidad, ya que desde hace varias décadas, la administración de justicia en Colombia se ha caracterizado por la permanente congestión de los despachos judiciales, por el bajo rendimiento en el trámite de los procesos, por la mala distribución geográfica de los recursos y por una inadecuada planeación sectorial (García, 2006; Márquez, 2007). Como resultado de esta situación, se ha generado un ambiente de impunidad y de poca credibilidad en el sistema judicial, que ha tenido como repuesta una serie de reformas a los códigos, que no han solucionado la crisis.

En síntesis, aún persisten, entre otros, los siguientes problemas: a) La desconfianza hacia los hombres que imparten la justicia; b) la legitimación de la justicia respecto a la credibilidad y viabilidad en la aplicación de las normas; c) la ausencia de definiciones judiciales claras y justas; d) el aumento y altas tasas de reincidencia de los delitos, lo que además representa sobrecarga de expedientes; e) precaria asignación de recursos humanos, tecnológicos y económi-

\footnotetext{
${ }^{4}$ Las investigaciones encontraron que miembros de las fuerzas de seguridad de Colombia perpetraron un número significativo de ejecuciones extrajudiciales en un patrón que se fue repitiendo a lo largo del país. Aunque estos asesinatos no fueron cometidos como parte de una política oficial, se encontró que muchas unidades militares comprometidas con los llamados "falsos positivos", en los cuales las víctimas eran jóvenes asesinados por militares, a menudo por beneficio o ganancia personal de los soldados. Generalmente, las víctimas fueron atraídas bajo falsas promesas por un "reclutador" hasta una zona remota donde eran asesinadas por soldados que reportaban que había "muerto en combate" y tomaban medidas para manipular y encubrir la escena del crimen". Dentro de las Fuerzas Armadas, el éxito fue medido por el número de guerrilleros muertos y promovido por un entorno en el que hubo poca o ninguna rendición de cuentas. Los soldados sabían que podían quedar impunes (Informe de Naciones Unidas 2009, sobre Ejecuciones Extrajudiciales, 98.5\% de impunidad http://www.extrajudicialexecutions.org/dhcolombia / Martes 25 de mayo de 2010).
} 
cos; f) procesos costosos y dispendiosos; g) poca relación entre la pena y el daño causado a las víctimas; $h$ ) las penas no cumplen con su papel de resocialización y rehabilitación (ingreso de la persona a la sociedad); i) abandono de la(s) víctima(s) en los procesos judiciales en Colombia; j) hacinamiento en reclusión de menores; k) la mayoría de normas no se basan en estudios ni proyecciones de las instituciones académicas e investigativas (Rivera y Barreto, 2008).

Estos argumentos sobre la crisis en la administración de justicia, la situación de la niñez y adolescencia en el contexto colombiano, las alarmantes estadísticas sobre los menores víctimas e infractores de la ley, las nuevas tendencias en la justicia de responsabilidad penal de adolescentes, el panorama internacional respecto a la justicia restaurativa y la nueva implementación en la legislación colombiana y específicamente en la ley 1098 de 2006 se convierten en argumentos suficientes para llevar a cabo esta investigación sobre ¿Cómo se están aplicando los criterios de justicia restaurativa para atender casos de responsabilidad penal para adolescentes en el Centro de Servicios Jurídicos Especiales para Adolescentes (CESPA) en la ciudad de Bogotá y los municipios de Cundinamarca- Colombia?

\section{Objetivos del estudio}

\section{Objetivo General}

Analizar la aplicación de los principios de justicia restaurativa para atender casos de responsabilidad penal para adolescentes en el CESPA en la ciudad de Bogotá y los municipios de Cundinamarca, Colombia.

\section{Objetivos específicos}

- Identificar los conocimientos y experiencias que tienen los funcionarios del CESPA Bogotá y Cundinamarca sobre la aplicación de los principios de justicia restaurativa en casos de responsabilidad penal para adolescentes.

- Valorar la opinión que tienen los funcionarios sobre la oportunidad de diseñar un programa de justicia restaurativa en casos de responsabilidad penal para adolescentes en Colombia.

- Identificar los procedimientos y requerimientos para la implementación de un programa de justicia restaurativa en caso de responsabilidad penal para adolescentes en Colombia.

\section{Metodología}

Esta investigación es de corte cualitativo con un diseño de carácter exploratorio-descriptivo que se ajusta a los presupuestos teóricos-conceptuales de los principios de justicia restaurativa descritos en la revisión bibliográfica. Para el estudio se definieron algunas categorías iniciales de orden deductivo, pero durante el proceso de recolección, sistematización y análisis de la información surgieron nuevas categorías inductivas y de agregado. Las categorías permitieron organizar los resultados en las unidades de análisis, de modo que la información obtenida cumpliera con los requisitos de ser exclusiva y excluyente para el análisis, la discusión y la presentación de resultados.

\section{Participantes del estudio}

El trabajo ha contado con una muestra intencional integrada por treinta profesionales que trabajan en el CESPA, pertenecientes a: Policía Judicial, Fiscales delegados, Jueces de Conocimiento, Jueces de Control de Garantías, Defensor de Familia, Ministerio Público y Policía de Infancia y Adolescencia.

\section{Anexo}

Se diseñó y aplicó una entrevista en profundidad basada en un cuestionario de 20 preguntas que se dividieron de acuerdo con las categorías del estudio de la siguiente forma:

- Cinco preguntas para obtener información sobre los conocimientos y la experiencia que tenían los funcionarios sobre la aplicación de la ley 1098 de 2006. 
- Ocho preguntas sobre el conocimiento respecto a lineamientos internacionales y nacionales en justica restaurativa.

- Seis preguntas que indagaban por programas y procedimientos específicos en justicia restaurativa que se estuvieran aplicando en el CESPA de la ciudad de Bogotá y municipios de Cundinamarca.

- Una pregunta acerca de los recursos institucionales con que se cuenta para desarrollar programas de justicia restaurativa.

\section{Resultados}

Los resultados de la investigación se dan a conocer a partir de las categorías del estudio previamente establecidas y que permitieron organizar la información aportada por los grupos de profesionales que trabajan en el CESPA de la ciudad de Bogotá y en los municipios de Cundinamarca.

En primera instancia se establecen las diferencias y coincidencias en el desarrollo de la Ley de infancia y adolescencia entre los participantes del estudio, y lo pertinente al tema de responsabilidad penal de los adolescentes teniendo como aspecto central el desarrollo de programas de justicia restaurativa.

Como resultado general se observa que existen diferencias en cuanto al conocimiento y la experiencia de los profesionales que laboran en el CESPA de la ciudad de Bogotá y los profesionales del CESPA de los municipios de Cundinamarca. Los primeros han recibido mayor capacitación y formación en cuanto el Código de Infancia y Adolescencia.

\section{Resultados obtenidos en la categoría de conocimiento y experiencia}

La información arroja como resultado que el grupo de Jueces de CESPA de Bogotá en comparación con los profesionales de CESPA Cundinamarca cuentan con una mayor experiencia, por cuanto empezaron a implementar el sistema desde el año 2006 con mayor cantidad de casos atendidos; mientras que en el caso de los profesionales de Cundinamarca, algunos de ellos no han recibido capacitación y comenzaron a aplicar la ley el primero de junio de 2009 con un número reducido de casos. Los profesionales del CESPA de Bogotá muestran mayores conocimientos que han adquirido a partir de cursos institucionales, del interés particular de los funcionarios, por la necesidad de resolver los casos atendidos tratando de cumplir con los principios del derecho internacional humanitario, la convención de los derechos del niño, los principios de Beijing, las directrices de Riad, la Constitución Política de Colombia, aspectos del derecho procesal y el Código de niños, niñas y adolescentes.

El grupo de profesionales que participaron en el estudio, le dan un valor importante al nuevo carácter pedagógico, diferencial y específico de la norma, para que ello sea posible, previamente el Estado colombiano debe cumplir con su responsabilidad de generar las condiciones necesarias para el buen desarrollo físico, psicológico, social de los niños, las niñas y los adolescentes. Además, ofrecer las condiciones mínimas y oportunidades socio económicas para las familias de los adolescentes infractores de la ley, acompañándolos con políticas públicas efectivas hacía las madres cabeza de familia, quienes no cuentan con los suficientes recursos económicos, ni apoyos institucionales para el cuidado, la crianza y la supervisión de los hijos.

Otro punto, es que consideran que hay deficiencias en la planeación y ejecución de la misma ley, debido al poco tiempo que ésta lleva en el Sistema de Responsabilidad Penal de Adolescentes. Critican los tiempos que otorga la norma para la sanción de los adolescentes, ya que según ellos, es muy limitado, y no se logra un proceso pedagógico, ni de resocialización adecuado, lo que provoca que los adolescentes sistemáticamente se burlen de las sanciones legales.

Posteriormente centran sus críticas en las políticas públicas en materia de educación, ya que según los profesionales existe una alta deserción escolar de los adolescentes infractores de la ley. Hacen referencia a las escasas instituciones educativas especializadas en los problemas de los adolescentes en la ciudad de Bogotá y los municipios de Cundinamarca, que por ser tan escasos, también generan preocupación por la ubicación tan distante teniendo consecuencias en una grave separación del adolescente de su entorno familiar. 
Además, consideran los profesionales del CESPA, que falta mayor apoyo y seguimiento de los casos para conocer la situación en que se encuentra el adolescente, su familia y el entorno donde viven, ya que esta labor no se puede cumplir por el número escaso de profesionales con los que se cuenta actualmente y que se requiere de una mejor formación en las diferentes áreas del conocimiento. Critican a la vez, la falta de coherencia en la unificación de criterios de actuación profesional e interprofesional, al no contar con protocolos especializados dirigidos a: la prevención, atención, remisión y toma de decisiones de los profesionales que atienden los casos. En esta misma línea, plantean problemas sobre los criterios que les permita articular las funciones de las instituciones educativas frente a los profesionales las familias y el propio adolescente.

Sobre las instituciones de la institución familiar, consideran que hay un escaso compromiso de las familias para cumplir el papel pedagógico de la sanción, objetando el papel de los padres respecto a las pautas de crianza, la ausencia de normas, y caracterizando a los padres como negligentes y en ocasiones cómplices de las conductas delictivas de sus hijos. Existe además, un grave abandono de las figuras paternas en el cumplimiento de responsabilidades, pues dejan a cargo de sus hijos únicamente a la madre y la familia extensa de ésta en la mayoría de los casos.

Frente a las medidas pedagógicas de los adolescentes, consideran que tienen cada vez más problemas con la justicia a más temprana edad, y que la mayoría de adolescentes no tiene conciencia para reparar a sus víctimas; los adolescentes sólo se allanan a los cargos para que no sean remitidos a las instituciones.

Al indagarse por los principios de la ley sobre bienestar, tutela y resocialización, la mayoría de funcionarios coinciden que se intenta cumplir con los derechos de los y las adolescentes infractores de la ley, así como hacer cumplir los derechos de las víctimas. La mayoría de participantes consideran que se ha mejorado la vigilancia y control para que no se vulneren los derechos de los adolescentes cuando intervienen los profesionales pertenecientes a la Policía de infancia y adolescencia, al Instituto Colombiano de Bienestar Familiar, la Fiscalía General de la Nación y los Juzgados.
Respecto al tema de la resocialización vuelven a invocar los mismos problemas para hacer posible las sanciones pedagógicas y algunos mencionan que en pocos casos se han logrado experiencias exitosas, y éstas se asocian a las siguientes circunstancias: son delincuentes que delinquen por primera vez, buena crianza, compromiso de la familia, suficiente tiempo para hacer supervisión, delito leve, consciencia y voluntad de cambio por parte del adolescente.

Hay consenso entre los profesionales al decir que si bien la Ley 1098/06 establece unos parámetros para materializar los principios y que, además, éstos hacen lo posible por cumplir la norma, la realidad demuestra que no existe una relación clara entre la doctrina legal, los aspectos metodológicos y los propósitos buscados para la resocialización, que es el fin menos logrado. De acuerdo con las respuestas obtenidas, lo anterior se atribuye principalmente a un sistema desbordado por la cantidad de casos, la carente disposición por parte del menor infractor, la escasa corresponsabilidad de la familia, el entorno social y cultural, las deficiencias legales, la falta de infraestructura y los pocos recursos económicos.

Sobre el carácter pedagógico, diferencial y específico que exige la norma, los profesionales la encuentran como una norma valiosa y fundamental que permite: a) Procesos reflexivos de los adolescentes infractores en torno al compromiso de no reincidir en las conductas delictivas; b) dar un trato diferencial respecto a los adultos por su inmadurez psicológica; c) entenderlo como una sanción pedagógica y no punitiva alejándolo de las instituciones de los adultos; d) cada infracción debe tener un tipo específico de sanción pedagógica dependiendo de la gravedad de los hechos, las características y necesidades del adolescente.

\section{Resultados obtenidos en la categoría sobre principios de justicia restaurativa}

En cuanto a la aplicación de los principios de justicia restaurativa, los funcionarios del CESPA tanto de Bogotá como de los municipios de Cundinamarca, a excepción de un juez (conocimiento), un fiscal y un defensor de familia, admiten no tener conocimientos ni experiencia sobre el tema, pero 
asocian la aplicación de estos principios con: a) El procedimiento dirigido a restablecer los derechos de las víctimas y a buscar una indemnización económica; b) al incidente de reparación integral; c) a restablecer los vínculos sociales que afectan la conducta del adolescente infractor; d) la solicitud de perdón por parte del victimario y la iniciación de un proceso de rehabilitación para la víctima; e) se asocia con los principios de Justicia y Paz de Estado Colombiano; f) probar un delito para reparar a la víctima; g) tratar de que el adolescente reflexione buscando un mínimo de convivencia y comprendiendo que el menor infractor también ha sido víctima de la crisis social; h) evitar al máximo que los adolescentes sean institucionalizados; i) un procedimiento que se aplica a los delitos asociados con hurto y lesiones personales; j) la indemnización, el perdón y las garantías de no repetir la conducta; k) habilitar y dar protagonismo a las víctimas a través de acciones como la asistencia psicológica, la información adecuada, un trato amable y coherente con la situación denunciada.

Las respuestas que se obtienen de la pregunta si los problemas de criminalidad en adolescentes pueden ser abordados desde la perspectiva de la justicia restaurativa, algunos funcionarios responden que es posible cuando: a) se cuente con el grado de corresponsabilidad social entre el Estado, la comunidad, la familia, la víctima y el adolescente infractor; b) las necesidades básicas de alimentación, salud, educación, vivienda, y empleo estén satisfechas y garantizadas; c) existan oportunidades para los adolescentes y se logre la transformación del entorno que los lleva a delinquir; d) se comprendan los contextos y las necesidades del adolescente que vive en Bogotá y los que viven en los municipios de Cundinamarca.

Los profesionales del CESPA critican la falta de apoyo por parte del Estado para implementar programas de justicia restaurativa, creen que se debe brindar mayor asistencia psicológica y social, así como ampliar la participación de otras entidades. Además, se hace necesario un seguimiento más riguroso de este proceso que permita medir la reincidencia de los adolescentes infractores y verificar el cumplimiento de los acuerdos. Los participantes en la investigación piensan que este último aspecto incide en la presentación continua de conciliaciones que no resuelven el conflicto, sino que, por el contrario, lo mantienen en un círculo vicioso, determinando la poca credibilidad que los menores infractores tienen de las instituciones.

Los beneficios de los programas de justicia restaurativa son: a) Permite tomar conciencia a edades temprana; b) integra conceptos de distintos profesionales con un mismo fin que es garantizar el bienestar del menor e impartir justicia c) la victima siente que hace parte de un Estado social y democrático de derecho por medio de la reparación; d) el Estado y la sociedad cambia la percepción del adolescente como un delincuente; e) continúa el empoderamiento del adolescente como un sujeto de derechos y responsabilidades; f) el victimario piensa en la condición con la victima; g) los padres toman conciencia de la falta de autoridad, cuidado y disciplina respecto a sus hijos; h) la victima tiene la posibilidad de conocer la verdad; i) se ahorra el desgaste de la ley; j) la víctima y el victimario participan de manera conjunta en la solución del caso; k) Se cumple la función pedagógica que pretende los estándares internacionales; 1) se aleja la idea de impunidad reparando a la víctima y reinsertando al adolescente a la sociedad; $\mathrm{m}$ ) se evita la congestión de los procesos judiciales, solucionándolos de forma rápida e integral. Otros funcionarios interpretan los beneficios de la justicia restaurativa como una ventaja para que los menores no sigan delinquiendo.

Ante la pregunta sobre las principales críticas del modelo de la justicia restaurativa, los profesionales responden que: a) Este enfoque se excede en garantías y protección a los adolescente infractores, por lo tanto facilita la reincidencia de sus conductas; b) no se ha contado con la inducción ni capacitación necesaria para los profesionales y el equipo técnico que interviene sobre estos casos; c) la reparación que piden las víctimas no es proporcional al daño causado, y tampoco se ajusta a las condiciones económicas del adolescente o en su defecto de la familia; d) no todos los delitos son susceptibles de aplicar a este modelo; e) el modelo no se ajusta a los diferentes contextos y necesidades sociales; f) ha presentado dificultades la aplicación de los modelos de justicia restaurativa porque se mantienen muchos de los principios de la justicia punitiva y retributiva; g) este modelo requiere de importantes transformaciones y 
prácticas culturales que permitan una mayor cohesión social que apoye a la justicia; h) el adolescente no asume la responsabilidad de los hechos cometidos, simplemente tiene llamados de atención, y quienes reparan son los padres; i) aprovechando la flexibilidad del modelo de justicia restaurativa, los adolescentes se convierten en un instrumento de los adultos para la comisión de delitos; j) el exceso de protección del modelo de justicia restaurativa genera para el adolescente un perjuicio de adaptación social en su vida adulta; $\mathrm{k}$ ) el modelo de justicia restaurativa no regula la posibilidad de que los adolescentes presenten altos índices de reincidencia y mayor gravedad en los delitos cometidos; 1) hay confusión por parte de algunos funcionarios respecto a la etapa procesal donde se debe aplicar la justicia restaurativa; $\mathrm{m})$ algunos funcionarios no tienen críticas porque consideran que hasta el momento se están implementando los programas de justicia restaurativa.

\section{Resultados obtenidos en la categoría de programas de justicia restaurativa}

Sobre la implementación de los programas de justicia restaurativa los profesionales hacen las siguientes consideraciones: a) Se debe contar con una capacitación integral frente al tema, que incluya además de los profesionales a todas las partes involucradas en el proceso (familia, comunidad y colegios); b) se requiere hacer una mirada más preventiva a partir de la Policía de Infancia y Adolescencia, la Fiscalía General de la Nación, el Instituto Colombiano de Bienestar Familiar, las familias y las instituciones educativas; c) se deben integrar los medios de comunicación para divulgar y socializar la importancia de la justicia restaurativa; d) hay que tener claridad sobre las funciones que debe cumplir cada uno de los profesionales, teniendo en cuenta el dominio y la especialidad de su disciplina, con el fin de lograr acciones articuladas y coordinadas frente a los programas de justicia restaurativa; e) es necesario contar con más recursos humanos, logísticos, técnicos y económicos para la implementación de programas de justicia restaurativa.

A la pregunta, ¿cómo debería operar un programa de justicia restaurativa?, los profesionales hicieron las siguientes consideraciones: a) Fortalecer los equipos técnicos e interdisciplinarios para desarrollar los programas; b) trabajar conjuntamente con el adolescente, la víctima, la familia y la comunidad a través de actividades culturales, académicas, recreativas, y promoviendo oportunidades laborales para lograr cambios en el sistema de justicia y la sociedad; c) hacer uso del principio de corresponsabilidad entre la sociedad, la familia y el Estado para atender al menor infractor; d) desarrollar estudios interdisciplinarios e interinstitucionales con el fin de establecer criterios para la viabilidad de los programas de justicia restaurativa; e) los programas de justicia restaurativa deben autorregularse a través de procesos de evaluación, lo que permitiría los cambios pertinentes para mejorar los procedimientos y los objetivos que se buscan.

En cuanto a la participación de la comunidad, los funcionarios del CESPA de la ciudad de Bogotá y municipios de Cundinamarca, consideran que hace falta más información sobre las alternativas de participación sobre los programas de justicia restaurativa. Para los profesionales del CESPA, el personal que trabaje con un enfoque enmarcado en la justicia restaurativa debe tener una capacidad de comprensión y compromiso con los usuarios y la problemática que allí se atiende. Consideran necesaria una capacitación especial para atender este tipo de conflictos desde una perspectiva pedagógica, ya que por su condición de defensores de familia, trabajadores sociales, psicólogos, policías de infancia y adolescencia, según señalan, no han sido preparados a profundidad para ello. En su opinión, hay una necesidad de recibir capacitación en áreas como asistencia social, negociación y resolución de conflictos y psicología infantil.

En general, los funcionarios señalan que la naturaleza de los conflictos de los adolescentes infractores de la ley hace imprescindible la participación coordinada entre jueces, fiscales psicólogos, trabajadores sociales, representantes de policía, de la Defensoría Pública y del Instituto Colombiano de Bienestar Familiar, así como la conformación de equipos interdisciplinarios que posibiliten la acción conjunta para restablecer los derechos y reparar a las víctimas. En suma, la realización de un trabajo intersectorial, interinstitucional e interdisciplinario. 


\section{Resultados sobre la categoría de recursos institucionales}

Para el cumplimiento de su tarea, los profesionales del CESPA de Bogotá y Cundinamarca señalan que hacen falta recursos y herramientas tales como instalaciones adecuadas, instrumentos tecnológicos, personal capacitado y espacio físico para la realización de talleres y la ejecución de entrevistas en las que se mantenga la privacidad. Así mismo, mencionan la falta de compromiso de las diferentes entidades y del apoyo de las instituciones relacionadas con la problemática del adolescente.

En síntesis, la aplicación de cada una de las categorías propuestas en el estudio, ponen en evidencia un desconocimiento sobre los procedimientos generales de los programas y mecanismos de justicia restaurativa por parte de las instituciones tanto judiciales como educativas, así como de los profesionales, la comunidad, la familia y los adolescentes infractores.

\section{Conclusión}

La aplicación de cada una de las categorías propuestas en el estudio, ponen en evidencia un desconocimiento sobre los procedimientos generales de los programas y mecanismos de justicia restaurativa de las instituciones tanto judiciales como educativas, así como de los profesionales, la comunidad, la familia y los adolescentes infractores en principios y programas de justicia restaurativa. Una buena política criminal en el SRPA debe permitir mecanismos de transformación cultural, social, comunitaria, familiar e individual para interiorizar las formas de justicia restaurativa. Se hace indispensable cumplir con el principio de corresponsabilidad entre el Estado, la comunidad, la familia, las víctimas y adolescentes infractores para hacer viable los principios y programas de Justicia Restaurativa en Bogotá y los municipios de Cundinamarca. Por último, el estudio muestra la necesidad de generar criterios de actuación profesional e interprofesional a partir de protocolos especializados dirigidos a: la prevención, atención, remisión y toma de decisiones de los profesionales que atienden los casos. En esta misma línea, plantean problemas sobre los criterios que les permita articular las funciones de las instituciones educativas frente a los profesionales las familias y el propio adolescente.

\section{Referencias}

Álvarez, M. (1999). Vivencias y derechos: el adolescente trasgresor de la ley penal, Instituto de Estudios del Ministerio Público de la Procuraduría de Bogotá, Bogotá, Colombia.

Álvarez. M., Corzo, L., Mendoza, V., Parra, S. y Rodríguez, M. (2008) Semillas de cristal. Sistema de responsabilidad penal para adolescentes, alcances y diagnóstico. Instituto de Estudios del Ministerio Público de la Procuraduría de Bogotá, Bogotá, Colombia, 2008.

Brito, D. (2009). La Justicia Restaurativa. Comunidad que construye paz. Diseño de un modelo. Santiago de Chile: Editorial Universidad Bolivariana.

Carranza, E. Tiffer, C. y Maxera, R. (2002). La reforma de la justicia penal juvenil en América Latina, documento elaborado para la XI Sesión de la Comisión de las Naciones Unidas sobre Prevención del Delito y Justicia Penal, ILANUD, abril 2002.

Código de la Infancia y la Adolescencia. Ley 1098/ 2006. 29 de agosto de 2006 Gaceta del Senado. Año XV N 376.

Consedine, J. (2002). La tradición celta: reparando el daño. En J. Consedine, (Ed.). La justicia restaurativa sanando los efectos del crimen. Colombia. Consejo Episcopal Latinoamericano.

Constitución Política de Colombia de 1991. 10ª ed., Temis.

Convención sobre los Derechos del Niño. (1989). Resolución 44/25, 20 de Noviembre de 1989. Recuperado de http://www2.ohchr.org/spanish/ law/crc.htm

De la Cuesta, J. (1998). Líneas directrices de un nuevo derecho penal juvenil y de menores. En Eguzkilore No 2. País Vasco España.

Fiscalía General de la Nación (2007). Principio de Oportunidad I. Módulo de formación para Fiscales. Bogotá, D. C.

Fundación Restrepo Barco (2004). Recuperado de http://www.armada.mil.co/?idcategoria $=44965$

García, V. (2006). Justicia, mercado y democracia: Un examen crítico de las teorías económicas 
sobre el (in)cumplimiento del derecho y su relación con el desarrollo, la justicia y la democracia. En R. Uprimny, C. Rodríguez y M. García (Eds.) ¿Justicia para todos? Sistema judicial, derechos sociales y democracia en Colombia, 49-107. Bogotá: Norma.

Gimenez, E. (1999) "La conciliación víctima delincuente como alternativa a la justicia penal", en M. A. Soria, (comp.). La víctima: entre la justicia y la delincuencia. Aspectos psicológicos, sociales y jurídicos de la victimización. Barcelona: PPU.

Gómez, J. (2008). ¿Subsidiariedad? ¿Eso con qué se come? En Infraestructura en Acción, 4, 2-3. Recuperado de http://www.dps.gov.co/documentos/3147_Magazin_de_Infraestructura_Nov_200 8.pdf

Griffiths, C. y Hamilton, R. (1996). Sanctioning and Healing: Restorative Justice in Canadian Aboriginal Communities. En B. Galaway y J. Hudson (Eds.), pp. 175-191. Restorative Justice: International Perspectives, Monsey, NY: Criminal Justice Press/Willow Tree Press.

Instituto Colombiano de Bienestar Familiar (2010). El ICBF invertirá este año 1.5 billones de pesos en nutrición infantil. Colombia: Author.

Larrauri, E. (2004). Tendencias actuales de la justicia restauradora. En F. Pérez (Ed.), Serta In Memoriam Alexandria Barrata, (pp. 439-464). Salamanca: Universidad de Salamanca.

Márquez, A. (2007). La justicia restaurativa versus la justicia retributiva en el contexto del_sistema procesal de tendencia acusatoria. Revista Prolegómenos. Derechos y Valores de la Facultad de Derecho. 10 (20), 201-212. Recuperado de http://www.umng.edu.co/docs/revderecho/rev2de 2007/13.JusticiaRestaurativa.pdf

Ministerio de Educación Nacional (2009). Recuperado de http://menweb.mineducacion.gov. co/seguimiento/estadisticas/inicio.php

ONU (2002). Comisión de prevención del delito y justicia penal. Consejo económico y social Viena 16 a 25 Abril de 2002.

ONU (2009). Informe sobre Ejecuciones Extrajudiciales, $98.5 \%$ de impunidad. Recuperado de http:// www.extrajudicialexecutions.org/dhcolombia
Padilla, A. (2010). Tendencias y realidades del derecho en el siglo XXI. Capítulo 11. Tendencia y realidades en la justicia de menores. Editorial Pontificia Universidad Javeriana.

Pratt, J. (1996). Restorative Justice: International Perspectives. Colonization, power and Silence: A history of indigenous justice in New Zeland Society. En B. Galaway y J. Hudson (Eds.), (pp. 137156). Restorative Justice: International Perspectives. Monsey, NY: Criminal Justice Press.

Restrepo, O. (2007). Situación de la violencia juvenil en Cali. Programa DESEPAZ Cali, Colombia.

Rivera, S. y Barreto, L. (2008). Resumen ejecutivo la impunidad en el sistema penal acusatorio en Colombia. Recuperado de http://www.mij.gov.co/ econtent/library/documents/DocNewsNo4362Do cumentNo2463.PD

Rodríguez, L., Padilla, A., Rodríguez, L S. y Díaz, F. (2010). Criterios para un programa piloto de justicia restaurativa orientado a la atención de casos de violencia intrafamiliar en el Centro de Atención Integral a Víctimas de Violencia Intrafamiliar (CAVIF) de la Fiscalía General de la Nación en la ciudad de Bogotá, Colombia. Anuario de Psicología Jurídica, 20, 71-82.

Tiffer, C. (2000). Ley de justicia penal juvenil dentro de los modelos teóricos de política criminal y fuentes legales. En M. González M. y C. Tiffer (coord.). De la arbitrariedad a la justicia: adolescentes y responsabilidad penal en Costa Rica. (pp. 91- 181). Costa Rica, San José: UNICEF.

Varona, M. (1998). La mediación reparadora como estrategia de control social. Una perspectiva criminológica, (pp. 1 y 135). Granada: Comares.

Villegas, M. (2009). ¿Qué es el principio de intervención mínima? Revista Internauta de Práctica Jurídica, 23. 1-10. Recuperado de http:// www.ripj.com/art_jcos/art_jcos/num23/Principio. pdf

Von Hirsch, A., Ashworth, A. y Shearing, C. (2003). Specifying Aims and Limits for Restorative Justice: A "Making Ammends" Model?. En A. Von Hirsch, J. Roberts, A. Bottoms, K. Roach, y M. Schiff. (Eds.), Restorative Justice and Criminal Justice. Oxford: Hart Publishing.

Manuscrito recibido: 01/03/2012 Revisión recibida: 27/04/2012

Aceptado: 30/04/2012 